\title{
Information System as a Tool for Marine Spatial Planning: the SmartSea vision and prototype
}

\author{
Ari Jolma ${ }^{1}$, Ville Karvinen ${ }^{1}$, Markku Viitasalo ${ }^{1}$, Riikka Venesjärvi ${ }^{2}$, and Jari Haapala ${ }^{3}$ \\ ${ }^{1}$ Marine Research Centre, Finnish Environment Institute, Helsinki, Finland \\ ${ }^{2}$ Fisheries and Environmental Management Group, University of Helsinki, Kotka, Finland \\ ${ }^{3}$ Marine Research Unit, Finnish Meteorological Institute, Helsinki, Finland
}

\begin{abstract}
Planning the use of marine areas requires support in allocating space to particular activities, assessing impacts and cumulative effects of activities, and generic decision making. The SmartSea project studies the Gulf of Bothnia, the northernmost arm of the Baltic Sea, as resource for sustainable growth. One objective of the project is to provide an open source and open access toolbox for marine spatial planning. Here we present a vision for the toolbox and an initial prototype. The vision is based on a model of information system as a meeting point of users, information providers, and tasks. A central technical requirement for the system was found to be a data model and related database of spatial planning and a programmable map service. An initial prototype of the system exists comprising a database, data browser/editor, dynamic tile map service, web mapping application, and extensions for a desktop GIS.
\end{abstract}

Keywords: Marine Spatial Planning · Planning Support System · Information environment $\cdot$ Web Mapping $\cdot$ Dynamic Tile Service $\cdot$ WMTS

\section{Introduction}

Marine Spatial Planning (MSP) is a recently coined term for "a public process of analyzing and allocating the spatial and temporal distribution of human activities in marine areas to achieve ecological, economic, and social objectives that are usually specified through a political process" [1]. The aim and challenge of MSP is to manage both increasing and competing sectoral needs, especially for energy and natural resources, and increasing interest in maintaining biodiversity and conservation of nature [2]. The European Union (EU) has recently (2014) issued a Maritime Spatial Planning Framework Directive, which sets minimum common requirements for national MSP. EU has also launched a website for sharing experience and expertise in $\mathrm{MSP}^{1}$.

Effective MSP should be integrated and bring industries, such as fisheries, aquaculture, tourism, marine biotechnology, ocean or offshore energy, seabed mining, and commercial marine transportation, together to make coordinated decisions about how to use the sea and marine resources sustainably [1]. Some of these industries are

\footnotetext{
${ }^{1}$ http://www.msp-platform.eu

adfa, p. 1, 2011.

(c) Springer-Verlag Berlin Heidelberg 2011
} 
strictly in competition, while some may co-exist in same space. There are also complex interactions between marine, coastal, and terrestrial industries and environment.

Several plans concerning sea areas have been carried out or are in progress in Europe. Jones et al [2] describe 12 cases that were studied in the EU-FP7 project MESMA. Stelzenmüller et al [3] introduce a framework for monitoring and evaluation of spatially managed areas (SMAs), which was tested on nine of these case studies. Katsanevakis et al [4] introduce and analyzes ecosystem based marine spatial management as a holistic approach that could be supported by MSP.

Stelzenmüller et al [5] reviewed software tools that could have potential to support MSP and then developed a set of prototype tools to support MSP. They defined three categories of tasks that a practical MSP tool should support: (i) allocating space to particular activities, (ii) assessing impacts and cumulative effects of activities, and (iii) generic decision support. Zoning (partitioning the planning region into zones that are designed to allow or prohibit certain activities) is a popular method for managing both spatial interaction and cumulative effects [6]. However, in marine areas boundaries of zones are difficult to identify and enforce [5]. Also, zoning may result in a sectoral approach, which has been criticized [2].

Geodesign denotes a stated aim of developing plans, that is, synthesizing new geospatial data, with software tools [7]. Collaborative decision-making and networking is often emphasized in the development of geodesign tools [8-10]. For example, online tools like MarineMap ${ }^{2}$ and SeaSketch ${ }^{3}$ allow user participation and proposing of solutions, which can be compared. Merrifield et al [8] describe a spatial decision support system that enables stake-holder participation in designing Marine Protected Areas (MPAs) and Janssen et al [9] describe a method and a case of collaborative marine spatial planning involving the use of a decision support tool.

SmartSea is a research project (2015-2018), which aims to provide science-based guidance and new innovations for sustainable use of the marine resources of Finland. Finland is a Baltic Sea country and most of its foreign trade takes place by it. Finland is relatively poor on domestic energy sources and the shallow coastal waters of the Baltic Sea present an opportunity to develop offshore wind power. Due to its history, closedness, and current pressures, the Baltic Sea is a unique, fragile, and threatened sea area. Its environmental problems are, for example, eutrophication, lack of oxygen in the bottom layers, and the accumulation of environmental toxicants. The species diversity of the Baltic Sea is very low compared to oceans or lakes and rivers. Brackish water, young geological age of the basin, and the lack of certain habitats, such as tidal zone, cause the low biodiversity.

The SmartSea project will focus on Gulf of Bothnia (GoB), the northernmost arm of the Baltic Sea. GoB is divided into exclusive economic zones of Finland and Sweden. It is still a relatively undeveloped sea area with an area of $117000 \mathrm{~km}^{2}$.

The aim of this paper is to present the vision for the SmartSea MSP toolbox and describe its first prototype. The MSP toolbox will build on and be free and open

\footnotetext{
${ }^{2}$ www.marinemap.org

${ }^{3}$ www.seasketch.org)
} 
source software and provide open access to existing and new information resources and tools.

\section{SmartSea MSP toolbox: the Context}

The data that will provide input to marine spatial planning in GoB is extensive. A large amount of useful data already exists and a large portion of that is already openly available either as web mapping sites and/or downloadable datasets. The data is usually developed and maintained by national and international institutes. Especially, the Baltic Marine Environment Protection Commission (HELCOM), an intergovernmental cooperation body, collects, maintains, and provides a significant database on various aspects of the Baltic Sea. The data can broadly be divided into designated areas and boundaries; physical, chemical, biological, and ecological data describing the sea areas and species living there; and data about human activities.

An important data collection effort regarding the management of Finnish coasts and seas is the collaborative Finnish Inventory Programme for the Underwater Marine Environment (VELMU), where a large amount of data about underwater environment (e.g. abundance of keystone species and locations fish nursery grounds) has been collected since 2004. In VELMU, species specific data is extracted from the observations, such as underwater videos, and organized into geospatial layers, which are further used for species distribution modeling and other purposes. VELMU data is published on a web-mapping platform ${ }^{4}$.

Other examples of data that is available or that is being collected and developed for the project are wind conditions ${ }^{5}$, areas of importance for fisheries (surveys are being done by the Natural Resources Institute Finland), climate scenarios (developed by the Swedish Meteorological and Hydrological Institute), and the current and projected future oceanographic conditions (modeling and simulations carried out by the Finnish Meteorological Institute).

\subsection{Spatial Data Infrastructure}

Spatial Data Infrastructure (SDI) is a framework in which geographic data and metadata is made available for users and tools. When a connection between spatial data and its users is or is not a SDI is a debatable question but several characteristics has been given by researchers and practitioners. These include the use of specific technologies, such as the Internet, standards for data exchange and storage, policies for openness and other issues, and services for software applications and humans. The concept of Coastal/Marine SDI has been studied and promoted by the Global Spatial Data Infrastructure Association (GSDI) and the recently established Open Geospatial Consortium (OGC) Marine Domain Working Group.

\footnotetext{
${ }^{4} \mathrm{http}: / /$ paikkatieto.ymparisto.fi/velmu

${ }^{5} \mathrm{http}: / /$ www.tuuliatlas.fi/en/index.html is a website based on the wind data. It provides maps of average wind speed and estimated wind power production.
} 
The public SDI in Finland is based on INSPIRE (an EU SDI initiative) and implemented for example as a generic catalog ${ }^{6}$, a generic data browser ${ }^{7}$, and services maintained by individual institutes. A good example of what even a private individual can do with open marine data is the website aaltopoiju.fi, which presents current and historical meteorological and oceanographic data on a map interface.

\subsection{Collaborative modeling}

MSP requires collaborative modeling because its aim is to bring together relevant information from several sectors, and biological, geological, and oceanographic knowledge needs be integrated with information about human drivers. At each sector modeling is needed to produce data that is comprehensive and/or further developed/computed from the observation data. At the planning stage these data need to be integrated, which again requires modeling.

In SmartSea, the project partners bring in geological, oceanographic, biological, social, and economic models. The NEMO-Nordic ocean modeling framework [11] will be used to obtain water temperature, salinity, mean circulation, sea level, and ice extent scenarios in one nautical mile resolution. Simulations will be based on existing data from years 1980-2010 and the future conditions will be based on climate scenarios for the years 2030-2060.

\section{$3 \quad$ An information system for MSP}

MSP is a series of undertakings by planners and eventually decision makers to organize human activities on a sea area. We shall call the planners and decision makers as the 'users' of the information system for MSP. Goals of MSP comprise conflict minimization, ensuring viability of businesses, maintaining sustainability of the marine environment, and achieving maximal social benefits. Users need data, information, and knowledge together with social values, criteria, and goals. We shall call these the 'materials' of the information system. The users obtain the materials from data providers, science community, and various stakeholders, who we shall collectively call 'providers'.

The information environment (Figure 1.) has been defined [12] as the aggregate of individuals, organizations, or systems that collect, process, or disseminate information; and the information itself. The users' information environment dictates how well they can carry out their planning and decision making tasks. The state-of-the-art information environment in MSP is multifaceted: use of diverse Internet sources, official organizational data channels, elicitation of expert knowledge and stakeholder values, and personal efforts. Organization of these materials for planning, so that they can be used together in personal or organization information systems or software tools depends on they being in suitable formats. Otherwise manual data conversions and/or

\footnotetext{
${ }^{6}$ http://www.paikkatietohakemisto.fi

${ }^{7}$ http://www.paikkatietoikkuna.fi
} 
processing is required. Use of specific planning method tools usually requires lengthy data collection and preprocessing efforts. The private or local information environment can also greatly differ from the information environment shared among national, and especially international, MSP.

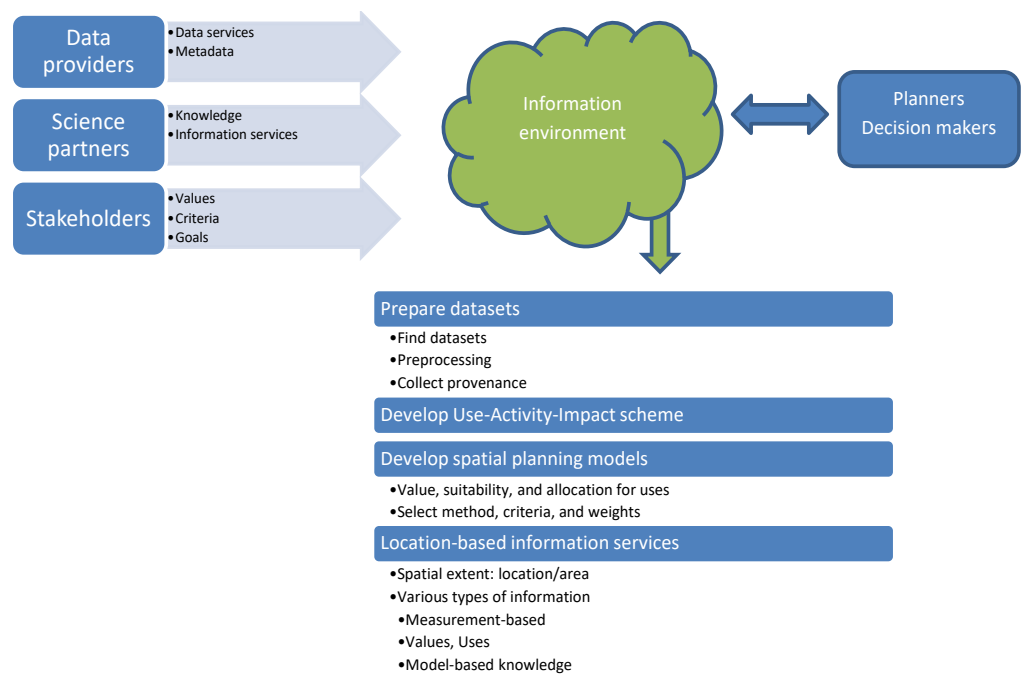

Fig. 1. Information environment as a place where data and information from providers become available for users with needs.

The promises and goals of spatial data infrastructures, such as the European INSPIRE, has been multiple [13]: datasets are collected and kept at one location, where it originates or where it can be maintained most effectively; different data can be combined seamlessly together; data can be obtained at varying scales and levels, depending on the type of investigation at hand; and information about availability, usability, and constraints of data is easily available. While there has been progress towards these goals, it is still a challenge to bring existing data into MSP information environment due to reasons such as data size and format; and lack of suitable data or other service.

Typically, MSP requires new, specific datasets to be developed. These can be original datasets obtained by field research, or created with models of natural systems or human preferences. We see challenges in bringing these into the MSP information environment due to semantic problems created by differences in the user and provider communities, data interpretation requirements, and the level of collaboration required.

There are typically several software systems available or in operation in an information environment. Software systems need, in order to work efficiently, interoperability, which may take various forms and is one of the aspects of software architecture. MSP is, like planning in general, an activity where information is continuously added and processed until the result is satisfactory. The information environment is 
unbounded in this sense. Thus flexibility towards new information sources, and support for provenance become key properties of the overall system.

\section{$4 \quad$ A Vision for the SmartSea MSP toolbox}

MSP is a process that implements risk-averse ecosystem-based management of coastal and marine areas into practice, and enables integrated, forward-looking and consistent decision-making [1,5]. In addition, it is an instrument whereby relevant stakeholder groups can be involved in a transparent manner. In MSP, planners assess the cumulative effects of human pressures and seek to make the use of marine resources more sustainable and proactively minimize the conflicts between sectors. SmartSea aims is to provide information to MSP and to develop scientifically sound tools for risk analysis and decision-making.

Gulf of Bothnia, the initial spatial context of the MSP toolbox spans six administrative regions in Finland. Regions are the first administrative division below the country itself. A region has a large autonomy in spatial planning, which adds a dimension to the overall national MSP.

Traditionally, MSP is based on static environmental data. However, considering ongoing climate change, any long-term plans should consider projected changes in the environmental parameters. In the GoB, the most apparent changes will be the increasing sea temperature, reducing ice cover, and decreasing salinity. All these will have considerable impacts on marine biota from plankton to fish species but they will also have an impact on fish farming and other marine related businesses and their possibilities.

The suitability of an off-shore location for activities depends on oceanographic, like wave height, sea level, sea surface temperature, and ice loads and geological conditions, like seabed substrate. A desirable feature of an MSP toolbox is thus to be able to consider return periods of extreme events and their characteristics.

The fundamental requirements for the SmartSea MSP toolbox have been identified as supporting identifying what could and what should not be done in various areas of the region, what would be the value and impacts of the activities, and where specific activities should be placed. These involve collection, analysis, and integration of spatial data that is relevant to possible uses of sea areas, information about gained benefits and ecological impacts of activities, and a consideration for shared use of areas.

Generic requirements for the SmartSea MSP toolbox are visualization of spatial data, both independently and as overlays to detect spatial competition. Modeling can be partially offline but for ad hoc type of queries and plans interactive use of some models will probably be needed. Design capabilities are needed for creating plans. Supporting collaborative and participatory planning requires tools for analyzing competing plans, capabilities for shared use, and visualization.

In a previous project ${ }^{8}$ we developed a demonstration MSP tool. The tool was based on a workflow, where pressures from human activities and the current environmental

${ }^{8}$ Transboundary tools for spatial planning and conservation of the Gulf of Finland (TOPCONS) http://www.merikotka.fi/topcons/ 
state were first coded on spatial rasters of the planning region. Second, the competing plans were devised and their local and spatially distributed impacts were computed and stored as compatible rasters. In the third and final step, valuation weights were computed for impacts and the plans could be ranked based on a resulting single indicator value for each plan. The local and spatial impact and valuation computations were based on models implemented as Bayesian Networks. The tool was implemented as a QGIS (community developed free and open source GIS) ${ }^{9}$ plugin.

\section{Components of the SmartSea MSP toolbox}

The SmartSea MSP toolbox is planned as a distributed application with one server and two types of clients. A work-in-progress setup of services has been developed on a server computer in a cloud infrastructure. The server hosts a database and services that build on it. The database comprises spatial data and data supporting the planning process. The services can provide data for desktop software such as QGIS and for applications running in a web browser. A simple web browser application for visualizing sectoral values, zoning plans, and competition was developed. Its core functionality is based on a WMTS service, which can dynamically compute spatial layers from existing layers based on simple rules. A goal is to be able to describe the logic of computing the value and impacts of, stimulating, allowing, and/or denying activities in areas as functions of the characteristics of those areas and activities as a set of sequentially applied rules.

The source code for the toolbox is available at github ${ }^{10}$.

\subsection{The Database}

The database is implemented on a RDBMS (PostgreSQL+PostGIS) installation in addition to files in filesystem and it consists of three parts: the tool, the data catalog, and the datasets.

The tool part is based on a data model for planning, which consists of core classes Plan, Impact, Layer, and Rule (See Figure 2.). A plan is a comprehensive allocation of zones in the planning region. A zone is assumed to have a designed use, which is a realization of a designed use class, which may comprise several activities. An activity should be a well-defined description of a single enterprise or a ban of such ${ }^{11}$. Thus it is possible to compute both the benefits and the negative impacts of a use for an area. It is clear that the composition of this kind of uses is a large task in itself.

An (environmental) impact is something that an activity causes. The activity - impact mapping is done via an environmental pressure as the EU Marine Strategy Framework Directive instructs. In the quantitative assessment, an attempt is made to determine numerically the probabilities of different pressures and the likely extent of

\footnotetext{
${ }^{9}$ http://qgis.org

${ }^{10}$ https://github.com/ajolma/SmartSeaMSPTool

${ }^{11}$ The description may, depending on what kind of MSP policy is adopted, include temporal and other conditions and thus be quite complex in the end.
} 
the losses under a particular pressure. Several alternatives can be used to define the probability distributions attached to the pressures. These include, for example, statistical datasets, experimental data, modeling outputs, and expert knowledge. Expert assessments of relationships between activities, pressures, and impacts on ecosystem components have been obtained in other projects ${ }^{12}$. Structured expert judgement can be integrated into modeling approaches to improve predictions, and it can be particularly valuable if no relevant published data can be found. Further, as managers might believe that models do not result in better decisions than those supported by the opinions of experts, expert-informed modeling can contribute to bridging the gap between researchers and decision-makers [14, 15]. Experts give estimates for what pressures various activities create, what is their spatial range, how serious the impact of the generated pressure is to a range of ecological components, and how strongly the experts believe in their estimates. This data may be enough (assuming additional expert data is available, e.g., to account for cumulative impacts) to compute impacts of zoning proposals. However, this part of the project is still ongoing. Comprehensiveness of this kind of data is of course an issue since both the range of possible activities and ecological components is very wide. Based on the obtained results from the impact assessment, a decision analysis between the demand for marine resources and conservation of nature values will be performed.

A layer is a spatial dataset, prepared by the system as a $20 \mathrm{~m} \times 20 \mathrm{~m}$ raster covering the planning region ${ }^{13}$. A layer represents an attribute (the layer class) associated with a use and it is obtained from a SDI or computed from datasets or other layers using rules. A rule is a mathematical expression for computing a layer from datasets or other layers. A rule consists of several elementary rules, which are combined in a specific way (the rule class). Rules can be combined exclusively, i.e., locations are deemed unsuitable for a use because of its characteristics; inclusively, i.e. locations are deemed suitable for some use because of its characteristics; multiplicatively, i.e., the value at a location is computed by multiplying values representing characteristics of it; or additively, i.e., the value at a location is computed by summing values representing characteristics of it.

\footnotetext{
12 These are mostly projects done within the HELCOM collaboration and include projects HOLAS II, TAPAS, and MARISPLAN.

${ }^{13}$ It is possible to create such a raster layer or input for one from a vector layer on-the-fly using rasterization.
} 


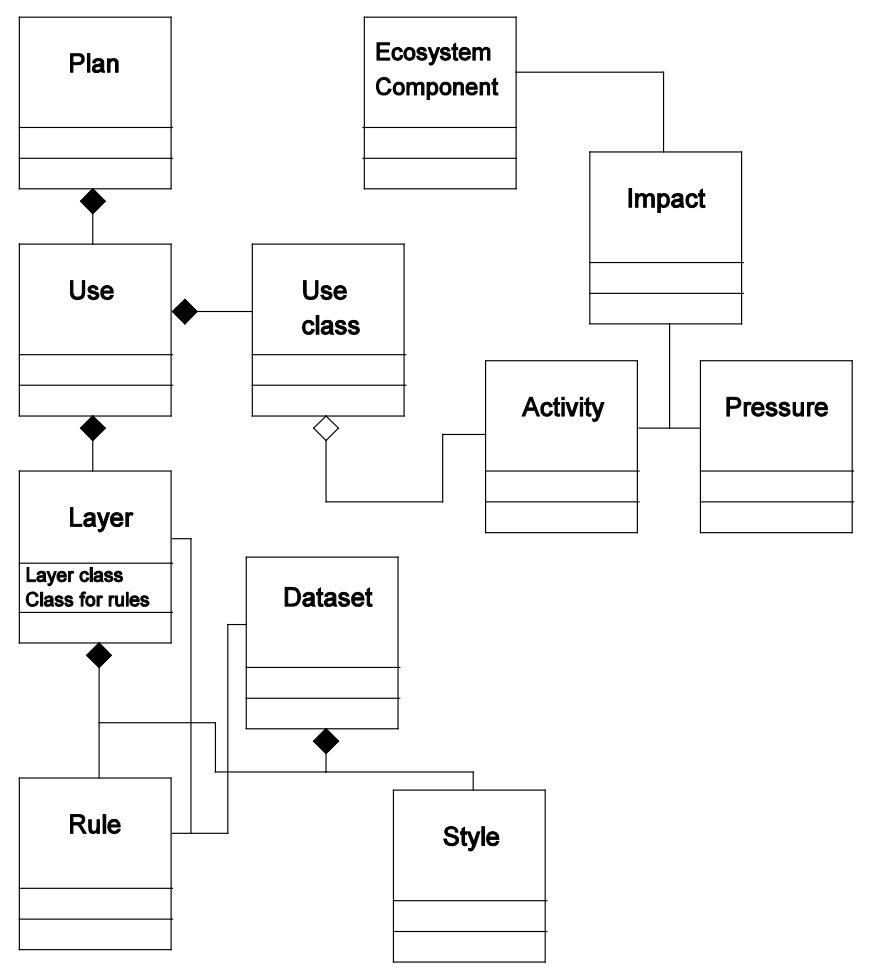

Fig. 2. A diagram of important classes and their relationships in the prototype MSP toolbox, see the text for explanations.

A layer can depict the value or benefit obtained from a specific activity or use, depict areas designated for a specific use or the suitability of locations for the use, or it can describe the impact of a use. The style is information how a dataset or a layer is visualized.

The data catalog is a simple database of metadata and provenance information that describes the datasets used by the planning tool. Its purpose is to document the origin of data and the workflow that was applied to prepare the data for the tool. The MSP toolbox will contain several datasets obtained and/or derived both from the national SDI and from research projects within the SmartSea umbrella and elsewhere. In order to retain this information and possibly reapply the workflow in the case of new versions of the original data come available, the provenance of the datasets must be kept.

\subsection{The Web Services}

Since the data is maintained on a server in a cloud, it is natural to provide an API to it as web services besides the native database interface and raw file download. The web services are divided into non-spatial and spatial services. The spatial services are 
OGC services and the non-spatial services are custom services providing JSON for applications and HTML for human users.

The spatial data services focus on WMTS (a raster tile service), which is ideal for creating responsive web mapping applications that need to cover large areas but also provide detailed views (using graduated zoom levels). In this project we defined a use case, where WMTS should provide layers that could be computed from several original layers using several rules and optionally the user should be able to edit the rules online. This clearly is not feasible using pre-computed layers. It also requires the concept of user in the service providing code so that it can use the correct parameter values for the rules when preparing tiles to the respective users. The current solution skips the capability document (thus the list of the available layers is not provided and the client program of the service must know how the layer names are constructed) but is able to compute layers very flexibly. The computation is based on coded layer names, where the code specifies the plan, the use, the type of layer, and the rules to use.

Another type of spatial service is one that is used to provide information from a selected location / area. The client sends the selected location or polygon area as WKT and information that tells, which (spatial) data to use for the reply, and based on those, the service computes a reply.

The non-spatial data services has two purposes: 1) to provide a document for applications, which lists the plans, uses, layers, and rules, that is, data to populate the GUI and also to construct WMTS requests (the layer names); and 2) to provide an interface to construct the plans, uses, and rules. The importance and complexity of this API was a finding for us in this research. The API is still developing since the database is developing but the overall structure is already quite stable. The construct API is a simple CRUD (create, read, update, delete) API and currently focuses on direct interactive use by humans and thus HTML. With this API the user is able to manage the objects in the database and their relations. The relationships are either associations (for example a use class is associated with one or more activities), compositions (for example a plan is composed of uses), or multiplicity (for example a use can have only one use class but multiple associated layers). The document API is there for client applications to obtain the tree-like data structure of plans, uses in them, layers in the uses, and the rules that are used to create the layers.

The web services were developed as Perl modules using the Starman web server ${ }^{14}$ and Perl modules related to it. The object-relational mapping is done using DBIx::Class ${ }^{15}$. The Perl Data Language (PDL) is used for raster computations and GDAL is used for spatial data access and management.

\subsection{The Web Mapping Application}

The focus of the user oriented development in the project has so far been on the web mapping application. The application is written in JavaScript using JQuery and

${ }^{14}$ http://search.cpan.org/dist/Starman

${ }^{15}$ http://www.dbix-class.org 
OpenLayers libraries and it is according to the MVC design pattern ${ }^{16}$. The application presents the user an interface that relies of the plan-use-layer-rule model of the planning problem (See Figure 3.). Additionally, the GUI contains a select location / area information tool.

The plan-use-layer-rule model upends the traditional GIS interface paradigm, which guides the user to construct maps from input data layers, which are presented. Here the result (the zoning or spatial allocation of uses) is what is presented to the user. Initially the whole region allocated for all uses and the paradigm is that the rules reduce (or add) the areas for uses.

The select location / area tool allows the user to select a location with a click or draw a polygon with a sequence of clicks. Once defined, the location or the polygon (as WKT) is sent to a server along with other selections made in the GUI, and the server reply is shown to the user. Currently this tool is a proof-of-concept only.

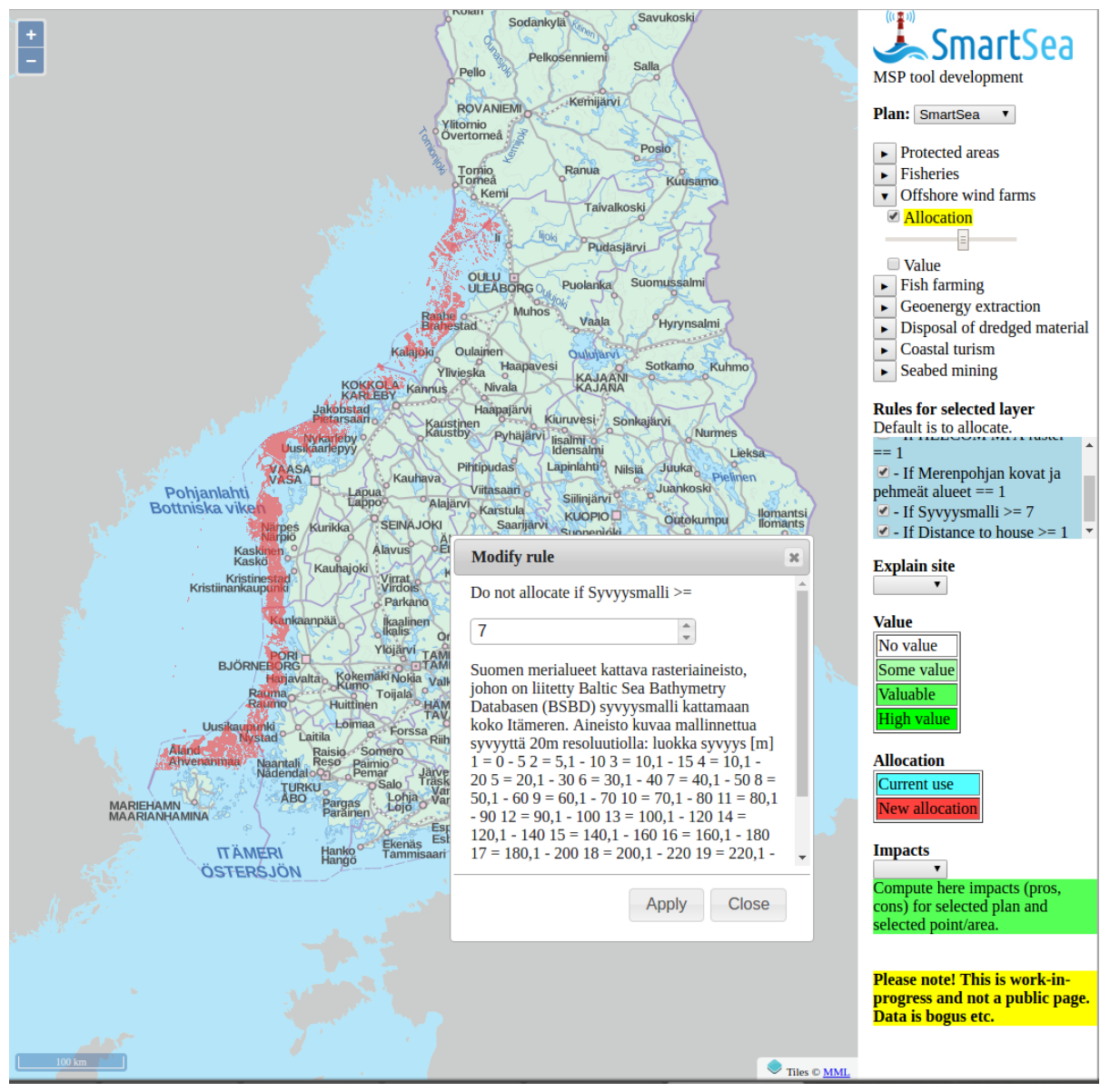

16 The code from https://alexatnet.com/articles/model-view-controller-mvc-javascript was used as a boilerplate. 
Fig. 3. A screenshot of the web mapping application prototype showing a selected plan (SmartSea), a visualized and selected computed allocation of areas for offshore wind farms (essentially a suggestion for manual design), and a dialog box allowing the user to edit one rule.

\subsection{The Desktop Tool}

Some of the tasks in MSP are unstructured and require generic tools capable of analysis and providing decision support. A desktop GIS is a typical generic tool for tasks that involve a spatial dimension. SmartSea project will develop open and freely available tools and QGIS was selected as the desktop tool for the SmartSea MSP toolbox. The goal is to develop a QGIS plugin, which integrates with the other components of the toolbox. Currently very little has been done to further this task.

Python is the main programming language for developing QGIS plugins and it is relatively easy to create one. In an initial test it was possible with a few lines of Python code to download the main GUI document in JSON from the web service and construct a tree of layers in the QGIS layer list in its GUI and download the WMTS layers. This is the first requirement since the WMTS server does not provide a layer list to choose from.

\section{Discussion}

MSP, despite being a novel topic, is a variation of the general theme of spatial planning. Numerous examples of how spatial planning can be supported with information technology exist.

Our vision for the SmartSea MSP toolbox is based on examples found in literature and our previous work. The toolbox will be based on an existing SDI but specific support needs to be built for representing zoning plans, impact computations, and negotiating between competing plans and sectoral needs.

The development of the application has so far been carried out completely within the research institute and all discussions related to its requirements and design have been among researchers. A public seminar organized by the Ministry of Environment announcing MSP in Finland was held in November 2016. The seminar revealed that only the administrative structure of MSP has been decided upon. The SmartSea project is a key endeavor towards supporting the actual planning. A series of workshops and collaboration will be organized for transferring knowledge and tools from the research community to the planning community.

INSPIRE is the directive defining the European SDI. One of its principles is that "it should be possible to combine seamless spatial information from different sources ... and share it with many users and applications" [16]. In our case the identified requirement to be able to dynamically compose planning proposals required us to download data from their sources to a dedicated server, which took care of the composition. This was in some cases necessary since the data was always not available as a service, or it needed some preprocessing. In those cases where the data was availa- 
ble as a service, moving it was considered a too fragile or slow operation to be a part of the MSP toolbox.

In a client-server environment for geospatial computing the map that is shown to the user can be rendered in the server or in the client from data sent by the server. If the data is dynamically changing, or, as in our case, the user has a great control over how the map is composed, the map needs to be generated dynamically. It is interesting that in the case of server side dynamically generated maps are usually considered only in relation to WMS and specifically not WMTS, which is seen as a set of statically rendered tiles ${ }^{17}$. In our case creating a plugin system for dynamically rendering raster tiles was easy since the WMTS code was developed by the main author.

MSP should be carried out with tools, which take account of uncertainties and are supported by a strong scientific background. We hope that the SmartSea MSP toolbox can eventually help to solve and structure complex and multidisciplinary problems, integrate knowledge and uncertainty, and communicate risks. Our approach identifies and quantifies explicit decisions among different objectives and represents spatial possibilities for sustainable blue growth. This work will be further developed to a decision analytic tool, which will provide a scientifically sound and consistent valuation method for evidence-based MSP.

Acknowledgements. We wish to acknowledge the funding received from the Strategic Research Council at Academy of Finland for the project SmartSea - Gulf of Bothnia as Resource for Sustainable Growth (Grant No: 292 985). We also acknowledge the editors of these proceedings and the anonymous reviewers for their work. Their comments significantly improved this paper.

\section{$7 \quad$ References}

1. Ehler C and Douvere F (2011) Marine Spatial Planning: a step-by-step approach toward ecosystem-based management, Intergovernmental Oceanographic Commission Manual and Guides No.53, UNESCO, Paris, France. http://unesdoc.unesco.org/images/0018/001865/186559e.pdf

2. Jones PJS, Lieberknecht LM, Qiu W (2016) Marine spatial planning in reality: Introduction to case studies and discussion of findings. Marine Policy 71: 256-264. doi:10.1016/j.marpol.2016.04.026

3. Stelzenmüller V, Breen P, Stamford T, Thomsen F, Badalamenti F, Borja A, BuhlMortensen L, Carlstöm J, D’Anna G, Dankers N, Degraer S, Dujin M, Fiorentino F, Galparsoro I, Giakoumi S, Gristina M, Johnson K, Jones PJS, Katsanevakis S, Knittweism L, Kyriazi Z, Pipitone C, Piwowarczyk J, Rabaut M, Kirk Sørensen T, van Dalfsen J, Vassilopoulou V, Vega Fernández T, Vincx M, Vöge S, Weber A, Wijkmark N, Jak R, Qiu W, ter Hofstede R (2013) Monitoring and evaluation of spatially managed areas: A generic framework for implementation of ecosystem based marine management and its application. Marine Policy 37: 149-164. doi:10.1016/j.marpol.2012.04.012

\footnotetext{
${ }^{17}$ See for example the topic "Dynamically drawn map services" in the Penn State University course GEOG 585 at https://www.e-education.psu.edu/geog585/node/697
} 
4. Katsanevakis S, Stelzenmüller V, South A, Kirk Sørensen T, Jones PJS, Kerr S, Badalamenti F, Anagnostou C, Breen P, Chust G, D’Anna G, Duin M, Filatova T, Fiorentino F, Hulsman H, Johnson K, Karageorgis AP, Krönke I, Mirto S, Pipitone C, Portelli S, Qiu W, Reiss H, Sakellariou D, Salomidi M, van Hoof L, Vassilopoulou V, Vega Fernández T, Vöge S, Weber A, Zenetos A, ter Hofstede R (2011) Ecosystem-based marine spatial management: Review of concepts, policies, tools, and critical issues. Ocean \& Coastal Management 54: 807-820. doi:10.1016/j.ocecoaman.2011.09.002

5. Stelzenmüller V, Lee J, South A, Foden J, Rogers SI (2013) Practical tools to support marine spatial planning: A review and some prototype tools. Marine Policy 37: 149-164. doi:10.1016/j.marpol.2012.05.038

6. Halpern BS, McLeod KL, Rosenberg AA, Crowder LB (2008) Managing for cumulative impacts in ecosystem-based management through ocean zoning. Ocean \& Coastal Management 51: 203-211. doi:10.1016/j.ocecoaman.2007.08.002

7. Dangermond J (2009) GIS: Designing Our Future, ArcNews, summer 2009.

8. Mitsova D, Wissinger F, Esnard A-M, Shankar R, Gies P (2013) A Collaborative Geospatial Shoreline Inventory Tool to Guide Coastal Development and Habitat Conservation. ISPRS Int. J. Geo-Inf. 2: 385-404. doi:10.3390/ijgi2020385

9. Merrifield MS, McClintock W, Burt C, Fox E, Serpa P, Steinback C, Gleason M (2013) MarineMap: A web-based platform for collaborative marine protected area planning. Ocean \& Coastal Management 74: 67-76. doi:10.1016/j.ocecoaman.2012.06.011

10. Janssen R, Arciniegas G, Alexander KA (2015) Decision support tools for collaborative marine spatial planning: identifying potential sites for tidal energy devices around the Mull of Kintyre, Scotland. Journal of Environmental Planning and Management. 58:4, 719-737. doi:10.1080/09640568.2014.887561

11. Hordoir R, Axell L, Löptien U, Dietze H, Kuznetsov I (2015), Influence of sea level rise on the dynamics of salt inflows in the Baltic Sea, J. Geophys. Res. Oceans, 120, 66536668, doi:10.1002/2014JC010642

12. Dictionary of Military and Associated Terms. S.v. "information environment." Retrieved 6 March 2017 from http://www.thefreedictionary.com/information+environment

13. [2] Directive 2007/2/EC of the European Parliament and of the Council of 14 March 2007 establishing an Infrastructure for Spatial Information in the European Community (INSPIRE). Retrieved 6 March 2017 from http://eur-lex.europa.eu/eli/dir/2007/2/oj

14. Addison PF, Rumpff L, Bau SS, Carey JM, Chee YE, Jarrad FC, McBride MF, Burgman MA (2013) Practical solutions for making models indispensable in conservation decisionmaking. Diversity and Distributions 19: 490-502, doi: 10.1111/ddi.12054

15. Aizpurua O, Cantú-Salazar L, San Martin G, Biver G, Brotons L, Titeux N (2015) Reconciling expert judgement and habitat suitability models as tools for guiding sampling of threatened species. Journal of Applied Ecology 52: 1608-1616, doi: 10.1111/13652664.12515

16. INSPIRE what if? Call for position papers for an ad-hoc workshop at the OGC TC meeting in Delft 23 March 2017. Retrieved 28 March 2017 from http://inspire.ec.europa.eu/sites/default/files/inspire_what_if._call_for_position_papers.pdf 